Published Version:

C. Dorn und V. Tacke (eds.), Vergleich und Leistung in der funktional differenzierten Gesellschaft, Wiesbaden: Springer VS, p. 41-71.

DOI:10.1007/978-3-658-17916-8_3

\title{
Rankings statt Stellenordnungen? Funktionen und Folgen von Leistungsvergleichen in internationalen Organisationen.
}

\section{Einleitung}

Der Vergleich nationaler Leistungsziffern ist im Falle internationaler Organisationen (IOs) keineswegs ein rezentes Phänomen. Schon der Völkerbund richtete eine statistische Kommission ein und veröffentlichte Jahrbücher, in denen die wichtigsten Zahlen zur Entwicklung der Mitgliedsstaaten aufgeführt wurden (Ward 2004, S. 36ff.). Das gegenwärtig Neue scheint aber zu sein, dass man für Leistungsvergleiche in IOs vermehrt auf die Form von Rankings zurückgreift. Die einschlägige Literatur behandelt solche internationalen Staaten-Rankings meistens als Steuerungsinstrumente der global governance, mit denen jenseits nationalstaatlicher Rechtssetzung und -sanktionierung die Befolgung von Normen mit schwach ausgeprägter Bindungswirkung (soft law) kontrolliert werden solle (siehe statt vieler: Davis, Fisher et al. 2012). Demnach werde die Umsetzung von vereinbarten Standards bzw. Zielen als Leistung beobachtet und in der Form von Rankings verglichen. Dies liefere Konkurrenzmotive und setze die Letztplatzierten einem erhöhten Begründungsaufwand gegenüber bestimmten Publika aus (Etzioni 1962; Friman 2010; Halliday 2012; Rotberg 2004). Statt formalisierter Sanktionen baue man daher auf die disziplinierende Wirkung des öffentlichen naming and shaming, bei welchem schlechte Rankingergebnisse zu Prestigeverlusten und damit verbundenen Folgekosten führen würden. Diese Beobachtungen sind sicher weitgehend berechtigt und konvergieren mit den Selbstbeschreibungen solcher Rankings (siehe etwa Kok 2004, S. 43). Die folgenden Überlegungen verstehen sich im Kontext dieser Literatur deshalb als Ergänzungsvorschlag und nehmen hierfür einen organisationssoziologischen Perspektivwechsel vor (siehe auch Kette und Tacke 2014). Der Ausgangspunkt dieses Beitrags liegt dabei zunächst in der Vermutung, dass Rankings für IOs die Möglichkeit bieten, eine fehlende Stellenordnung im Verhältnis zu 
ihren Mitgliedsstaaten zu substituieren. Rankings, so die These, bearbeiten dann Funktionen der Planung, Koordination und Kontrolle von Entscheidungsprämissen in IOs und kompensieren dadurch in bestimmten Hinsichten die Folgen einer fehlenden Stellenordnung.

Die Substituierbarkeit von Stellenordnungen und leistungsvergleichenden Rankings setzt voraus, dass sie unter einem bestimmten Gesichtspunkt als vergleichbar beobachtet werden können. Da hier demnach Vergleiche selbst als Vergleichsobjekte behandelt werden, liegt eine Bedingung der hier gewählten Vorgehensweise in der Annahme begründet, dass es sich bei Vergleichen um eine Form der Beobachtung handelt, die reflexiv auf sich selbst angewendet werden kann. Gleichwohl sollen hier nicht primär Rankings mit anderen Rankings verglichen werden, so wie man Käse mit Käse und Kreide mit Kreide vergleicht (Toulmin 1964, S. 354f.). ${ }^{1}$ Vielmehr sollen leistungsvergleichende Rankings mit organisatorischen Stellenordnungen verglichen werden, wobei der einheitliche Vergleichsgesichtspunkt nicht in der oberflächlichen Familienähnlichkeit der Vergleichsobjekte, sondern in einem gemeinsamen Funktionsbezug liegt, auf den hin Rankings und Stellenordnungen als austauschbare, funktional äquivalente Problemlösungen beobachtbar werden. Dabei soll mit der Systemtheorie eine Theorie fruchtbar gemacht werden, die ihre eigene Funktion in der Ermöglichung unwahrscheinlicher Vergleiche durch Abstraktion verortet (Luhmann 1992a, S. 408ff.). Damit korrespondierend wird eine dementsprechend funktional vergleichende Methode als Heuristik eingesetzt, die ansonsten sehr heterogene Sachverhalte aus einer kontraintuitiven Perspektive beleuchtet und nach Äquivalenzbeziehungen zwischen Problemlösungen sowie nach Folgeproblemen der Substitution von Problemlösungen fragt (Luhmann 1974a).

Ausgehend von der Beschreibung von IOs als Organisationen, die sich durch die Mitgliedschaft von Organisationen (nämlich Staaten²) auszeichnen, sollen Folgeprobleme identifiziert werden, die insbesondere in der prekären Autonomie von IOs im Verhältnis zu ihren Mitgliedsstaaten zu verorten sind (2). Diese Prekarität äußert sich u.a. auch darin, dass die Mitgliedschaft von Staaten in IOs nicht über die klassische Vorstellung zu besetzender organisatorischer Stellen vermittelt wird, sondern erst die

\footnotetext{
1 Den Hinweis auf das Chalk-and-Cheese-Principle bei Toulmin verdanke ich einem Vortrag von André Kieserling.

2 Ich verwende den Staatsbegriff der Einfachheit und Lesbarkeit halber und meine damit jenen Organisationenkomplex im Zentrum territorialpolitischer Systeme im Sinne von Wimmer (2000).
} 
Mitgliedsstaaten IOs konstituieren. Während die Stellenordnung in klassischen Organisationen weitgehend unabhängig von ihrer Umwelt variieren kann und damit einen wichtigen Ausdruck ihrer Autonomie darstellt, unterliegt das Verhältnis zwischen Mitgliedsstaaten und IOs wesentlich enger gefassten Bedingungen, die die Autonomiespielräume von IOs stark einschränken. In diesem Zusammenhang wird hier die These näher zu erläutern sein, dass Rankings die Funktion einer Stellenordnung als Äquivalente ersetzen und damit die Systemelastizität von IOs steigern können (3). Dabei wird davon ausgegangen, dass Rankings die Funktion von Stellenordnungen substituieren können, die in der flexiblen Koordination von organisatorischen Entscheidungsprämissen verortet wird. Daran anschließend werden Folgen von Rankings im Hinblick auf diese Entscheidungsprämissen in drei aufeinander folgenden Abschnitten zu diskutieren sein, in denen zuerst nach der Bedeutung von leistungsvergleichenden Rankings für die Mitgliedschaft in IOs gefragt wird, die dafür als Leistungsbeziehung charakterisiert werden soll (4). Daran anschließend wird die Koordinationsfunktion von Rankings im Verhältnis zwischen Mitgliedschaft und Entscheidungsprogrammen diskutiert, um zu zeigen, dass Rankings u.a. einen Problembezug zur Dosierung von programmierten Leistungsansprüchen an die Mitgliedsstaaten aufweisen (5). Neben der Entscheidungsprämisse der Mitgliedschaft und den Programmen von IOs wird auch nach Effekten von Rankings auf formelle und insbesondere informelle Kommunikationswege gefragt, um Möglichkeiten der Einflussnahme von IOs auf diese Kommunikationswege durch Institutionalisierung von Rankings zu rekonstruieren (6). Schließlich sollen die gewonnenen Einsichten kurz zusammengefasst werden, um in ein eher kritisches Resümee der Effekte von leistungsvergleichenden Rankings in IOs zu münden (7).

\section{Internationale Organisationen als Meta-Organisationen}

Die Formalisierung von Mitgliedschaftserwartungen in Form von Organisationen hat sich in der modernen Gesellschaft als ein überaus erfolgreicher Mechanismus erwiesen (Drori et al. 2006; Kühl 2014). Sie kann sogar rekursiv auf ihre eigenen Ergebnisse angewendet werden, sodass man mit Göran Ahrne und Nils Brunsson mittlerweile von Meta-Organisationen sprechen kann, also von Organisationen, die zu ihren Mitgliedern wiederum Organisationen zählen, welche die formalisierten Erwartungen der Meta- 
Organisation als Mitgliedschaftsbedingung anerkannt haben (Ahrne und Brunsson 2008). Meta-Organisationen sind freilich zunächst Organisationen wie alle anderen auch und damit gegenüber der Gesellschaft und Interaktionen auf einer eigenständigen Ebene sozialer Systemdifferenzierung angesiedelt (Luhmann 2015). Während man aber die Autonomie von Organisationen gegenüber der Gesellschaft und Interaktionen als weitgehend institutionalisiert voraussetzen kann, wird die Autonomie von MetaOrganisationen vor allem im Verhältnis zu ihren Mitglieder-Organisationen als besonders problematisch charakterisiert (Ahrne und Brunsson 2008, S. 110ff., 2012). Im Anschluss an diese Problembestimmung lassen sich dabei sachliche, soziale und zeitliche Bedingungen der Möglichkeit von Autonomie solcher Systeme näher spezifizieren (vgl. für das Folgende Luhmann 1974b, S. 156ff.):

- Die Autonomie von Meta-Organisationen äußert sich in der Sachdimension zunächst im Auseinanderziehen und im gegenseitigen Variabelhalten zweier Systemebenen: einer Ebene des Gesamtsystems, auf welcher die generalisierte wechselseitige Anerkennung der Meta-Organisation im Verhältnis zu ihren Mitgliedern vorausgesetzt werden kann, und einer Ebene des täglichen Kontakts und des konkreten Entscheidens. In Krisensituationen auf einer Ebene kann die andere Ebene die Führung übernehmen und das weitere Operieren der MetaOrganisation anleiten. Je höher der Grad der Generalisierung von Erwartungen in Meta-Organisationen gegenüber den Mitgliedsstaaten ist, desto flexibler kann auf der Ebene konkreter Entscheidungen agiert werden und desto eher steigt damit auch die Autonomie dieser Organisationen.

- In der Sozialdimension kann man davon ausgehen, dass eine wichtige Bedingung der Möglichkeit von Autonomie in einer hinreichend differenzierten Umwelt liegt. Die Meta-Organisation kann dann Abhängigkeit pluralisieren und kann dadurch verhindern, dass sie von einem Umweltsystem allein dominiert wird. Dabei liegt ein zuverlässiger Indikator für Autonomie in der Etablierung einer systeminternen Statusordnung, in der die Mitgliederorganisationen nicht ihren systemextern erworbenen Status in der Meta-Organisation geltend machen können, ohne ihn zuvor in eine systeminterne „Statuswährung“ konvertiert zu haben (Luhmann 1995, S. 160ff.). 
- Schließlich müssen Meta-Organisationen ihre eigenen Entscheidungsprämissen (d. h. Personal, Programme und Kommunikationswege) wiederum zum Gegenstand eigener Entscheidungen machen können und müssen, anders formuliert, in der Lage sein, autonom über Zeit zu verfügen und zu planen. Sie dürfen dann nicht lediglich Vollzugsorgane der Mitgliederorganisationen sein, die ohne weitere Entscheidungen Umweltinformationen unmittelbar in Systemoutput übersetzen, sondern sie müssen über einen eigenen Zeithaushalt verfügen, um Umwelteinflüsse in internen Entscheidungsprozessen verarbeiten zu können.

Die oben genannten und nach Sinndimensionen aufgetrennten Bedingungen der Autonomie von Meta-Organisationen lassen sich auf einer abstrakteren Ebene zusammenfassen als Problem der Aufrechterhaltung von Systemgrenzen, die angesichts einer sich ständig ändernden Umwelt invariant und gleichzeitig flexibel gehalten werden müssen. IOs können sich als Meta-Organisationen nicht vollständig gegenüber wichtigen Umweltsektoren (wie etwa ihren Mitgliedsstaaten) isolieren und dürfen andererseits nicht bloß Vollstreckungsorgane einzelstaatlicher politischer Entscheidungen sein. Sie müssen stattdessen eigene Strukturen und Routinen ausbilden können, die auch bei variierenden Umwelteinflüssen noch aufrechterhalten werden können (Luhmann 1971b, S. 117ff.). Es gilt also Offenheit und Geschlossenheit, Konstanz und Variabilität nicht nur füreinander flexibel $\mathrm{zu}$ halten und situativ sowohl Geschlossenheit und Konstanz, als auch Offenheit und Variabilität als Entscheidungsgesichtspunkte wählen $\mathrm{zu}$ können, sondern diese auch wechselseitig aneinander $\mathrm{zu}$ steigern. Einen der wichtigsten Modi, mit denen sich solche wechselseitigen Steigerungen erzielen lassen, bildet die Generalisierung bzw. die Abstraktion. Sie ermöglicht die Formulierung systeminterner Prämissen, die auch noch angesichts situativ stark variierender Umweltereignisse Orientierung bieten und entsprechende Erwartungen einrasten lassen können. Wie im folgenden Abschnitt zu zeigen sein wird, operieren auch Rankings und Stellenordnungen mit solchen Abstraktionen, die in IOs die gleiche Funktion bearbeiten können, sich aber in ihren konkreten Problemlösungen und Folgeproblemen unterscheiden. 


\section{Zur Äquivalenz von Stellenordnungen und Rankings in IOs}

Die Vorstellung einer „Stelle“ gehört für Organisationen zu den wichtigsten Formen der Generalisierung (Luhmann 2000, S. 231ff.). Eine Stelle ist zunächst nichts weiter als ein leerer Signifikant, „eine inhaltsleere Identität mit auswechselbaren Komponenten“ (ebd., S. 233). Sie fungiert als Scharnier, welches Entscheidungsprämissen derart aneinander koppelt, dass zwangsläufig geplante Änderungen an einer Prämisse mit anderen Prämissen koordiniert werden müssen. „Wenn Personen eingestellt werden, wird ihnen eine bestimmte Aufgabe zugewiesen, und das erfordert zugleich die Zuordnung zu bestimmten Abteilungen und bestimmten hierarchischen Ebenen. [...] Es ist kaum vorstellbar, dass Personen rekrutiert werden, ohne dass man ihnen bestimmte Tätigkeiten zuweist; so wenig, wie man sich vornehmen wird, eine Aufgabe zu erfüllen, ohne festzulegen, wer die entsprechenden Tätigkeiten ausführen soll“ (ebd., S. 232). Stellen verknüpfen allerdings nicht nur Entscheidungsprämissen miteinander, sondern konstituieren deren wechselseitige Substituierbarkeit und damit zugleich deren Vergleichbarkeit. Während eine Stelle als tertium comparationis konstant gehalten werden kann, können im Hinblick auf diese Stelle ansonsten verschiedene Bewerber als gleich behandelt und anhand einer ganzen Reihe von systeminternen Kriterien verglichen werden. Von einer Stelle aus gesehen, erscheinen aktuelle und potentielle „Stelleninhaber“ dann als substituierbar und eben dadurch: als vergleichbar. Man kann aber nicht nur das Personal als substituierbar behandeln, wenn es etwa gilt, bestimmte Programme unbedingt umzusetzen. Ebenso lässt sich auch das Personal als konstante Prämisse „einfrieren“, um zu sehen, welche variablen, substituierbaren Programme sich mit diesem Personal realisieren lassen. Insgesamt lässt sich daher ohne Übertreibung sagen, dass Stellenordnungen in Organisationen geradezu paradigmatisch das Problem der Herstellung von Substitutionsbeziehungen symbolisieren, welches letzten Endes auch das Bezugsproblem von Vergleichen schlechthin bildet.

\section{Mitgliedsstaaten als Stelleninhaber?}

Das bisher Gesagte gilt freilich auch für IOs: Sie besetzen Stellen mit Personal, welches sich an Kommunikationswege zu halten und programmförmig definierte Aufgaben zu bewältigen hat. Aber IOs haben es als Meta-Organisationen darüber hinaus mit Mitgliedsstaaten zu tun, die wesentlich schwieriger zu handhaben sind, die sich nicht ohne Weiteres in einer Stellenordnung zueinander in Beziehung setzen lassen und 
denen Aufgaben kaum arbeitsteilig zugewiesen werden können. Die Mitgliedschaft von Organisationen in Meta-Organisationen stellt also insofern einen besonderen Typus von Mitgliedschaft dar, der sich nicht nur darauf reduzieren lässt, dass man es nun mit Organisationen statt mit Personal zu tun hat ${ }^{3}$, sondern auch darin seine Eigenart hat, dass diese Form der Mitgliedschaft nicht über Stellen definiert wird: IOs schreiben keine kontingentierten Stellen für neue Mitgliedsstaaten aus, die es dann nach Maßgabe bestimmter Qualifikationen zu besetzen gilt, ebenso wenig wie Staaten sich auf offene Stellen bewerben, die durch einen genau umrissenen Aufgabenbereich und eine Zuordnung in einer zwischenstaatlichen Weisungshierarchie definiert wären. Dies bringt erhebliche Folgeprobleme mit sich: IOs können mangels einer Stellenordnung nur bedingt eine organisationsinterne Statusordnung im Verhältnis zwischen Mitgliedsstaaten formalisieren. Das schränkt sie in ihrer Autonomie gegenüber den Mitgliedsstaaten ein. Denn diese tragen ihren systemexternen Status in die Organisation hinein und können ihre eigenen Situationsdefinitionen und Selbstdarstellungen weitgehend ohne Rücksichtnahme auf organisationsinterne Prämissen durchsetzen. IOs können ihre Mitgliedsstaaten aber auch nicht durch Karrieren integrieren oder sie damit zur Leistung bzw. zur compliance motivieren. Darüber hinaus können sie auf der Ebene generalisierter Programme ohne eine Stellenordnung auch kaum flexibel und situationsadäquat zwischen ihren Mitgliedsstaaten differenzieren, sodass die Programme (mit allen möglichen Härten) für alle gleichermaßen gelten müssten - es sei denn, man verhandelt individuelle Lösungen mit Mitgliedsstaaten und konzediert explizite Ausnahmen. Allerdings werden dadurch Generalisierungseinschränkungen in Kauf genommen, die sogar die programmierten Regeln in IOs infrage stellen können. ${ }^{4}$ Schließlich werden ohne eine Stellenordnung die Mitgliedsstaaten von IOs auch nicht als substituierbar behandelt. Das kann durch eine simple Kontrollüberlegung überprüft werden: Hätte man es in Bezug auf Mitgliedsstaaten mit einer Stellenordnung zu tun, müsste sich nach dem Ausscheiden eines Mitgliedsstaates die Frage der Nachfolge auf der nun freigewordenen Stelle stellen. Aber am Beispiel der Europäischen Union kann man selbst bei den kontroversesten Debatten rund um „Grexit“ bzw. „Brexit“ beobachten, dass die jeweiligen Mitgliedsstaaten im Hinblick auf deren Mitgliedschaft

\footnotetext{
3 Aus diesem Grund wird hier statt der Entscheidungsprämisse „Personal“ in weiterer Folge von der Entscheidungsprämisse „Mitgliedschaft“ ausgegangen.

${ }^{4}$ Ein prominentes Beispiel stellt etwa der „Britenrabatt“ bei der Finanzierung des EU-Budgets dar. Vgl. Spence 2012.
} 
eben nicht als substituierbar (und damit: als vergleichbar) beobachtet werden, zumal die EU ihre Systemidentität maßgeblich ihren Mitgliedern verdankt: „The nature and efficacy of the EU is affected by the identity of its members. An EU in which France and the UK were replaced by Russia and the Ukraine would be a very different organization" (Ahrne und Brunsson 2005, S. 437). Stattdessen werden Individualität und Einzigartigkeit von Mitgliedsstaaten in einer Art und Weise beobachtet, die man beinahe als „Singulaphilie“ bezeichnen könnte, und die eng mit der Semantik der Nation verknüpft zu sein scheint ${ }^{5}$.

Singulaphilie bildet neben Stellenordnungen und Rankings somit ein weiteres funktionales Äquivalent für die Regulierung von Substitutionsbeziehungen: Behandelt man jeden Staat als Einzelfall, erübrigt sich Vergleich durch Stellen oder Rankings. Ein Folgeproblem liegt jedoch darin, dass IOs nicht ohne Weiteres Programme entscheiden können, um daraufhin jene Mitgliedsstaaten auszusuchen, die willig, fähig und hinreichend motiviert sind, diese Programme auch auszuführen, oder gegebenenfalls unkooperative Mitgliedsstaaten durch andere zu ersetzen. Mit Rankings verfügen IOs aber über eine Möglichkeit, Mitgliedsstaaten in bestimmten Hinsichten als substituierbar zu behandeln. Die Bedingungen dieser Möglichkeit verdienen eine nähere Erläuterung.

\section{Die „virtuelle“ Substituierbarkeit von Mitgliedsstaaten in Rankings}

Wie oben gezeigt wurde, müssen IOs im Regelfall von der konstanten Mitgliedschaft von Staaten ausgehen, um daraufhin Programme dahingehend zu vergleichen, ob sie von den Mitgliedsstaaten auch ausgeführt werden können. Die zentrale These dieses Beitrages lautet aber nun, dass IOs in der Form von leistungsvergleichenden Rankings ein funktionales Äquivalent für eine Stellenordnung realisieren und ihre Mitgliedsstaaten damit zwar auf einer eher „virtuellen“ Ebene, aber doch mit durchaus realen Folgen als quasi-substituierbar behandeln können. Sie können dadurch in einem gewissen Maße Flexibilität bei der Koordination ihrer Entscheidungsprämissen erreichen und damit auch ihre Autonomie steigern, wenngleich sie nicht alle Leistungen von Stellenordnungen substituieren können und mit gänzlich anderen Folgeprobleme

\footnotetext{
${ }^{5}$ Bei Herder heißt es hierzu bereits: „So modificiren sich die Nationen nach Ort, Zeit und ihrem inneren Charakter; jede trägt das Ebenmaas ihrer Vollkommenheit, unvergleichbar mit andern, in sich“ (Herder 1802, S. 385).
} 
umzugehen haben. Aber wie wird die Äquivalenz von Stellenordnungen und Rankings vermittelt, und welche Folgeprobleme ergeben sich aus dem Einsatz von Rankings?

Rankings können eine Stellenordnung unter der Bedingung ersetzen bzw. ergänzen, dass sie einen ähnlichen Bezug zum Problem der abstrakten Symbolisierung von auswechselbaren Identitäten aufweisen und daher auch für Koordinationsprobleme zwischen Entscheidungsprämissen eingesetzt werden können. Platzierungen in Rankings gleichen in gewisser Hinsicht der abstrakten Vorstellung einer Stelle: Die erste, zweite oder letzte Position in einem Ranking sind ebenso inhaltsleere Identitäten wie Stellen und lassen zunächst offen, womit diese Identitäten besetzt werden sollen. Eine Rangplatzierung verliert ihre Identität nicht, wenn sie in einem Jahr von Guatemala und im nächsten Jahr von Papua-Neuguinea besetzt wird. Rangplatzierungen sind in diesem Sinne ebenso Substitutions- und damit auch: Vergleichsgesichtspunkte wie Stellen. Sie haben darüber hinaus den Vorzug, dass es immer einen ersten, zweiten, dritten Platz usw. geben muss, wenn das Ranking etwas besagen soll, sodass nicht alle denselben Platz einnehmen können. Man setzt dafür häufig quantifizierbare Indikatoren ein, die sich dermaßen verfeinern lassen, dass sich Differenzen in der zweiten und dritten Nachkommastelle auf die Rangplatzierung auswirken können. Dadurch bleibt sichergestellt, dass jeder Rangplatz nach Möglichkeit mit nur einem Mitgliedsstaat besetzt wird. Mit leistungsvergleichenden Rankings können Mitgliedsstaaten also auf einer niedrigschwelligen Ebene als substituierbar behandelt werden, die aber nicht die Mitgliedschaft von Staaten selbst berührt. Der folgende Abschnitt soll daher die Beziehung zwischen IOs und ihren Mitgliedsstaaten zunächst als Leistungsbeziehung darstellen, um in den darauf folgenden Abschnitten $\mathrm{zu}$ fragen, wie leistungsvergleichende Rankings in IOs die Funktion der Kopplung und Koordination von Entscheidungsprämissen übernehmen können.

\section{Die Mitgliedschaft in IOs als Leistungsbeziehung}

Mit den Mitgliedsstaaten und dem Personal können in IOs zwei Mitgliedschafts-Typen voneinander unterschieden werden, die zunächst einen gemeinsamen Problembezug im Hinblick auf die Formalisierung von Erwartungen in IOs aufweisen. Das Personal muss die formalisierten Erwartungen der Organisation als Bedingung für Mitgliedschaft akzeptieren, so wie auch IOs die Anerkennung von Qualitäten und Leistungen ihres 
Personals durch die Aufnahme in die Organisation artikulieren. Ebenso müssen aber auch Mitgliedsstaaten die formalisierten Erwartungen der IO als Prämisse weiteren Verhaltens akzeptieren, so wie auch die IO zum Stichtag des Beitritts eines Mitgliedsstaates dessen Status quo in allen möglichen Hinsichten, vom Bruttoinlandsprodukt bis hin zur politischen Verfasstheit als Staat, anerkennt.

Der Status quo wird dann als Ausgangspunkt behandelt, von dem aus alles, was künftig verändert, entschieden, reformiert, also aus der Perspektive der IO Gegenstand künftiger Leistungen des Mitgliedsstaates werden soll, zunächst als Resultat von Geschichte akzeptiert wird. Damit wird auch hingenommen, was nicht geändert werden kann - um von dieser Lage aus kurzfristigere, kleinteiligere Verbesserungen und Reformleistungen erwarten zu können (Luhmann 1992b). Der Status quo verknüpft somit auf eigentümliche Weise Vergangenheit und Zukunft. Dabei interessieren weniger die konkreten Ereignisse und Prozesse, nicht die Selektionsgeschichte als solche, die zum Status quo geführt haben mag. Wichtiger scheint hingegen zu sein, dass sie als vergangene Leistung abstrahiert wird, etwa in der Form, wie auch die Vergangenheit von Geld abstrahiert werden kann: pecunia non olet. So wie auch im Falle des Geldes muss Leistung nur gegenwärtig im Hinblick auf eine offene, ungewisse Zukunft überzeugen. In dem Maße, in dem diese offene Zukunft die Führung im Erleben und Handeln gewinnt, muss die Vergangenheit aber hinreichend Anhaltspunkte für Erwartungen liefern, ohne die Zukunft zu determinieren. „Die Sicherheit, die eine unverrückbare Vergangenheit bot, muß [...] zum Teil aus der Zukunft selbst bezogen, zum Teil durch Garantien des Status quo geschaffen werden. Der Fortschrittsglaube selbst wird zum Sicherheitsäquivalent, das laufende Bestätigung braucht und in der Feststellung quantitativer Zunahme von irgend etwas auch findet" (Luhmann 1974c, S. 168). Hier erweist sich (wie auch bei Geld oder bei Schulnoten) Quantifizierung als ein Abstraktionsmedium par excellence. Sie abstrahiert vergangene Leistung derart, dass ihr zahlenförmiges Resultat (etwa in Form des BIP, der Ölförderquote oder der Arbeitslosenrate) auch im Hinblick auf mehrere mögliche Planungs- bzw. Zukunftsszenarien noch etwas besagen kann. Für den Beitritt eines Staates in eine IO muss deshalb im Regelfall belastbares Zahlenmaterial vorliegen, mit dem der Status quo dokumentiert wird.

Ein weiterer wichtiger Zukunftsbezug eines quantifizierten Status quo liegt in der Vermutung, dass er sich künftig steigern lässt. Er äußert sich u.a. dann „in der Form von 
Leistungsziffern, die überboten werden sollen oder jedenfalls nicht unterschritten werden dürfen" (Luhmann 1974c, S. 168). Es ist eben kein Status im Sinne einer konstant durchzuhaltenden Festlegung, sondern ein vorübergehender Status quo, der als Momentaufnahme in einem Konjunkturzyklus beobachtet wird. So wie man meint, aus guten Schulnoten Schlüsse über das zukünftig steigerungsfähige Lernpotential eines Mitarbeiters ziehen zu können oder aus Kontoauszügen die Kreditwürdigkeit eines potentiellen Schuldners herauslesen zu können, gilt auch in IOs für Beitrittskandidaten die Vermutung, dass das Fortschrittspotential an vergangenen Zahlen und Leistungsindikatoren ablesbar sei, sodass entsprechende Erwartungen gebildet werden. $^{6}$ Die Vergangenheit wird verdichtet, abstrahiert, quantifiziert und auf Konjunkturschwankungen hin beobachtet. Diese Form der Zeitbindung durch Leistungsziffern wird ganz im Sinne Pierre Bourdieus zum symbolischen Kapital. Es bestimmt maßgeblich darüber, ob und wie vergangene Leistung anerkannt wird, und ist damit eine Form von Kapital „mit kognitiver Basis, es beruht auf Erkennen und Anerkennen“ (Bourdieu 2004, S. 151).

Im konkreten Fall von IOs mag zwar die (An-)Erkennung vergangener Leistungen eine Rolle dabei spielen, ob ein Staat als Mitglied in Frage kommt oder nicht. Dennoch sind die basalen Kommunikationen in IOs nicht Erkenntnisse, sondern Entscheidungen, und Staaten werden nicht primär qua Erkenntnis über Qualitäten und Leistungen, sondern durch Entscheidungen zu Mitgliedern von IOs. Im Gegensatz zu Personalentscheidungen erfolgen aber, wie oben erläutert, Entscheidungen über die Mitgliedschaft von Staaten nicht über das Instrument der zu besetzenden Stelle. Im Falle der Vereinten Nationen etwa reicht die Anerkennung als Staat für die Mitgliedschaft aus, und es wird häufig erst durch die Mitgliedschaft in den Vereinten Nationen die Anerkennung von Staatlichkeit im weltpolitischen System symbolisiert (McNeely 1995). Viele IOs definieren sich darüber hinaus über einen regionalen oder ökonomischen Problembezug, der die Mitgliedschaft für eine Vielzahl von Staaten aufgrund deren geographischer Lage oder ökonomischer Entwicklung von vornherein ausschließt und nur ganz bestimmte Staaten in Betracht kommen lässt (vgl. Ahrne und Brunsson 2005, S. 438). Eine sekundäre Funktion des Stellenprinzips, nämlich Beitrittschancen für potentielle Mitglieder zu verknappen, greift dann im Fall von staatlichen Anwärtern nicht: Hier sind nicht die

\footnotetext{
${ }^{6}$ Espeland und Sauder (2007, S. 11f.) weisen darauf hin, dass die durch quantifizierende Rankings gebildeten Erwartungen sogar den Effekt von self-fulfilling prophecies zeitigen können.
} 
Stellen knapp, sondern die Zahl potentieller Mitglieder ist zu gering. Außerdem schließt die Mitgliedschaft eines Beitrittslandes die Mitgliedschaft eines anderen Beitrittslandes im Regelfall nicht aus. Es mag dann Rankings geben, mit denen Beitrittskandidaten unter verschiedenen Leistungsgesichtspunkten miteinander verglichen werden, und deren Ergebnisse mögen Reformanstrengungen motivieren oder in Beitrittsverhandlungen Munition für oder gegen einen Beitritt liefern. Aber da keine knappen Stellen zu besetzen sind, brauchen IOs keine priorisierenden Rankings, bei denen, wie in akademischen Berufungsverfahren, die Letztplatzierten allenfalls als Nachrücker beim Ausscheiden vorgereihter Kandidaten für eine Stelle in Frage kämen. ${ }^{7}$

Die Relevanz von Rankings für die Entscheidung über den Beitritt eines Staates zu einer IO dürfte sich in Anbetracht der hier genannten Bedingungen in Grenzen halten. Aber welche Rolle können Rankings nach einem Beitritt spielen, sobald es gilt, Entscheidungsprogramme in IOs umzusetzen? Diese Frage soll den Gegenstand des folgenden Abschnittes bilden.

\section{Rankings und Entscheidungsprogramme in IOs}

Im Allgemeinen können in Organisationen zwei Formen der Entscheidungsprogrammierung unterschieden werden (Luhmann 2000, S. 261ff.): Zweckprogramme legen Zwecke formal fest und ermöglichen dadurch eine weitgehende Freiheit bei der Wahl der Mittel, die zu deren Erreichung eingesetzt werden. Konditionalprogramme sind hingegen so gebaut, dass beim Vorliegen definierter Gründe bestimmte Entscheidungen $\mathrm{zu}$ erfolgen haben, ohne sie vorab festzulegen. In Organisationen lassen sich immer beide Formen der Programmierung beobachten, die einander wechselseitig bedingen und stützen. Im besonderen Fall von IOs gibt es aber im Verhältnis zwischen Mitgliedsstaaten und IO eine gewisse Präferenz für Zweckprogramme, da diese leichter und weniger konfliktanfällig zu institutionalisieren sind. Sie nehmen dann etwa häufig die Form von Zielen oder Standards an, zu deren Einhaltung sich die Mitgliedsstaaten gegenüber der IO bekennen, wobei sie aber weitgehend autonom darüber entscheiden können, ob und inwiefern die vorgegebenen Standards tatsächlich erreicht werden (Ahrne und Brunsson 2008, S. 124ff.; Brunsson

\footnotetext{
${ }^{7}$ In den Fortschrittsberichten der EU-Kommission über die Entwicklungen in Beitrittsstaaten werden zwar statistische Daten präsentiert, aber Rankings spielen faktisch keine Rolle. Siehe Europäische Kommission 2015.
} 
und Jacobsson 2002). Diese Reformansprüche an Mitgliedsstaaten können aber wiederum nicht im Kontext einer Stellenordnung formuliert werden, etwa in dem Sinne, dass programmierte Aufgaben definiert würden, die von einer bestimmten Stelle auszuführen wären. Man muss also andere Wege finden, um zu verhindern, dass Programme und Mitgliedsstaaten entkoppelt werden und unabhängig voneinander variieren. Die global governance-Literatur behandelt dieses Problem unter der Fragestellung, wie IOs ihre Mitgliedsstaaten zur compliance motivieren können. Argumentiert wird häufig, dass durch die Publikation von Rankings ein öffentlicher Druck aufgebaut werden soll oder dass Mitgliedsstaaten um die Gunst von Publika konkurrieren würden und sich deshalb in ihren compliance-Leistungen gegenseitig $\mathrm{zu}$ überbieten versuchen. Im Gegensatz zu dieser Literatur soll das Ausgangsproblem aber zunächst dahingehend neu gefasst werden, dass es nicht lediglich um compliance geht, sondern um die Frage, wie in IOs Entscheidungsprämissen zueinander in Beziehung gesetzt werden, wenn eine davon, nämlich die Mitgliedschaft von Staaten, als konstant vorausgesetzt werden muss und Mitgliedsstaaten nicht durch andere Staaten substituiert werden können. Dieses Problem der Kopplung von Programmen und Mitgliedsstaaten äußert sich unter solchen Bedingungen insbesondere als Problem der Dosierung von Leistungs- und Reformansprüchen, die an Mitgliedsstaaten adressiert werden, und kann in gewissen Hinsichten durch Rankings anstelle einer Stellenordnung bearbeitet werden. Die Folgen von Rankings für die Dosierung von Leistungsansprüchen sollen hier in vier Problemdimensionen (Überforderung/Unterschätzung, Generalisierung/Respezifikation, Anspruch/Rücksichtnahme, sowie Kurzfristigkeit/Langfristigkeit) näher bestimmt werden:

\section{Leistungserwartungen zwischen Überforderung und Unterschätzung}

Mitgliedsstaaten und IOs erhalten durch Rankings Richtwerte dafür, was politisch überhaupt leistbar ist, sodass der Horizont des Möglichen auf realistische (weil bereits realisierte) Grenzwerte eingeschränkt werden kann. Die (immer gegenwärtige) Zukunft eines Mitgliedsstaates liegt dann möglicherweise schon als Gegenwart eines anderen Mitgliedsstaates vor. Es können dann einerseits andernorts bewährte Programme imitiert werden, deren Umsetzung wiederum durch Leistungsvergleiche laufend kontrolliert werden kann, andererseits erhält man auf diese Weise ein mehr oder weniger wirksames Korrektiv für zu hohe Reformansprüche. 
Gleichwohl gibt es in der Weltgesellschaft regional stark variierende Bedingungen für politische Entscheidungen, sodass für Leistungserwartungen, die man an Afghanistan richten könnte, die Gegenwart Schwedens kaum als gegenwärtig denkbare Zukunft Afghanistans zu überzeugen vermag. Schraubt man Leistungserwartungen $\mathrm{zu}$ hoch, werden Enttäuschungen am laufenden Band produziert. Setzt man Leistungserwartungen $\mathrm{zu}$ niedrig an, könnten wichtige Potentiale und Ressourcen ungenutzt bleiben. Ohne jede weitere Anpassung von Leistungsansprüchen müssten IOs darüber hinaus Schweden und Afghanistan als auswechselbare Anwendungsfälle derselben Programme behandeln, die dann z.B. Entwicklungshilfegelder für Schweden vorsehen müssten. Werden hingegen nahe beieinanderliegende Rangplatzierungen zu Leistungsgruppen zusammengefasst (Werron 2012, S. 196), gelangt man nicht nur zu realistischeren Reformansprüchen, sondern kann für weniger stark variierende Bedingungen innerhalb solcher Leistungsgruppen dasselbe Programm entwerfen. Für den Problembezug der Koordination von Entscheidungsprämissen wären Ranglisten ohne die Bildung von Leistungsgruppen also zu detailliert, da nicht für jeden Rangplatz eigene Entscheidungsprogramme entworfen werden (Nullmeier 2004, S. 49). Eine weitere Problemdimension liegt daher in der Generalisierung von Leistungserwartungen, die aber auch im Einzelfall erfüllbar bleiben müssen.

\section{Leistungserwartungen zwischen Generalisierung und Respezifikation}

Leistungsgruppen sind im oben genannten Sinne Formen höherstufig generalisierten Sinns, mit denen die „Empfangselastizität“ einer IO so gesteigert werden kann, dass „das System ganz verschiedene Daten und Ereignisse als äquivalent behandeln kann [...]. Es braucht für vielfältige Ereignisse daher nur eine begrenzte Anzahl von Reaktionsmustern bereitzuhalten. Durch Änderung des Abstraktionsgrades seiner Aufnahmekategorien kann es die eigene Indifferenz gegen Unterschiede der Umwelt regulieren“ (Luhmann 1971b, S. 123). Leistungsgruppen, in denen mehrere Rangplatzierungen zusammengefasst werden, sind ein solcher Fall von systeminternen Abstraktionen, die konstant gehalten werden können und für sehr unterschiedliche Staaten wie Schweden, Korea und Island das gleiche Programm auslösen können, sofern sie in derselben Leistungsgruppe gereiht sind. Wie feingliedrig wiederum solche Gruppierungen erfolgen, kann flexibel an den jeweiligen Problembezug angepasst werden. So wie man auch in der Makroökonomie Populationen in Einkommensterzile, - 
quartile oder -quintile einteilt, lassen sich auch bei Rankings in IOs unterschiedliche Leistungsgruppen erstellen, die an das Erreichen bestimmter Indikatorenwerte oder Leistungsschwellen gekoppelt sind, oder durch schlichte Drei- oder Vierteilung der Rangliste gebildet werden. So wie die volkswirtschaftliche Statistik durch Einteilung in Einkommensgruppen zur Kontrolle politischer Entscheidungsprogramme (z.B. in Fragen der Besteuerung) beiträgt, können sich auch in IOs Programme an den Leistungsgruppen von Rankings orientieren. Sie müssen dann ihre Entscheidungsprogramme nicht an konkreten Staaten ausrichten (z.B. Entwicklungshilfe nur für die Staaten A, B, C), sondern können den Generalisierungsgrad von Rankings nutzen, um weitgehend unabhängig von Einzelfällen Programme an die Mitgliedsstaaten anzupassen und damit die Koordination von Entscheidungsprämissen der Mitgliedschaft und der Programmierung zu kontrollieren: Für die unterste Leistungsgruppe wird man z.B. Anpassungsbedarf für die entsprechenden Entwicklungshilfeprogramme der IO ablesen können, ohne sich bei der Programmierung auf bestimmte entwicklungsbedürftige Staaten festgelegt $\mathrm{zu}$ haben, und wird in der Frage flexibel bleiben können, welche Staaten künftig zu Anwendungsfällen eines solchen Programms werden. Eine andere, komplementär oder äquivalent genutzte Möglichkeit zur Bildung von Leistungsgruppen in Rankings bilden in dieser Hinsicht Gattungsbegriffe.

Auch Gattungsbegriffe sind eine Form höherstufig generalisierten Sinns und können für Planungsentscheidungen herangezogen werden. ${ }^{8}$ Für Staaten wird man insbesondere an die klassische Trias von Entwicklungs-, Schwellen- und Industrieländern, (mit weniger explizitem Zeitbezug) an die Unterscheidung von kleineren, Mittel- und Großmächten (Stern 2000, S. 102f.), an die Unterscheidung von erster, zweiter, dritter und vierter Welt oder an ältere Klassifikationssysteme in der UN-Bevölkerungsstatistik denken (Heintz 2012). Auch diese Gattungsbegriffe eignen sich für Planungsentscheidungen und für die Koordination von Entscheidungsprämissen, so wie etwa auch in Bildungseinrichtungen gattungsmäßig zwischen Kindern, Jugendlichen und Erwachsenen unterschieden wird. Für jede Gattung können dann bei gleichzeitiger Indifferenz gegenüber individuellen Eigenschaften der klassifizierten Objekte eigene Programme eingerichtet werden. Die genannten weltpolitischen Gattungsbegriffe lassen

\footnotetext{
8 Diesen Zeitbezug von (Gattungs-)Begriffen entnehme ich einem bislang unveröffentlichten Manuskript Luhmanns mit dem Titel „Zeithorizonte und Möglichkeitsvorstellungen der Planung“ (Luhmann o.J.). Für den Zugang zu diesem Manuskript danke ich Johannes F.K. Schmidt und André Kieserling.
} 
im Hinblick auf die gegenwärtige Zukunft offen, welche konkreten Exemplare man antreffen wird, wenn man z.B. nach Schwellenländern fragt; und sie können auf Inkonsistenzen bei der Koordination von Entscheidungsprämissen aufmerksam machen, wenn z.B. Entwicklungsländer (aus welchen Gründen auch immer) keinen Zugang zu zweck- und konditionalprogrammierten Fördergeldern erhalten.

Allerdings sind Gattungsbegriffe nicht das Resultat von Vergleichen, sondern der semantische Output einer klassifizierenden Subsumptionslogik. In diesem Sinne sind Klassifikationen, mit denen Mitgliedsstaaten konstante Qualitäten zugeschrieben werden, keine Vergleiche, sondern ein genau gegenläufiges Beobachtungsschema: Während Vergleiche potentiell offen sind und sehr heterogene comparata im Hinblick auf das tertium comparationis als substituierbar behandeln können, leisten Klassifikationen das Gegenteil, indem sie Vergleichs- und damit: Substitutionsschwellen hochmauern und Substituierbarkeit allenfalls innerhalb einer Klasse gleichartiger Objekte zulassen. Dennoch stehen Klassifikationen und Vergleiche in einem Verhältnis funktionaler Äquivalenz zueinander, da sich beide gleichermaßen auf das Problem der Regulierung von Substitutionsbeziehungen beziehen lassen. ${ }^{9}$ Außerdem werden in Rankings häufig Vergleiche und Klassifikationen miteinander verschränkt und bedingen einander. Rankings integrieren durch die Kombination von Klassifikation und Vergleich nämlich häufig sowohl konstante als auch variable Elemente: Einerseits vergleichen sie zeitlich variable Leistungen und geben vor, Leistungskonjunkturen von Mitgliedsstaaten in spezifischen Politikbereichen abzubilden, andererseits werden häufig jene Leistungsgruppen bzw. Gattungsbegriffe gebildet, bei denen erst in längerfristigen Zeitperspektiven mit der Änderung von Rangplatzierungen gerechnet werden kann.10 Die Zugehörigkeit zu einer Leistungsgruppe kann dann im Planungshorizont einer Entscheidung auch als konstant hinzunehmende Qualität behandelt werden, auf die bei Leistungsansprüchen gegenüber Mitgliedsstaaten Rücksicht genommen werden muss.

\footnotetext{
${ }^{9}$ Weitere funktionale Äquivalente wären etwa Intimität, Individualisierung (oder hier: Singulaphilie) oder die Konfundierung von Substitut und Substitutionsgesichtspunkt. In vormodernen Gesellschaften lässt sich letztere Option finden, bei welcher wechselndes „Personal“ ohne Vergleichsbeobachtungen substituiert und die zu besetzende „Stelle“ mit dem „Stelleninhaber“ verschmolzen wird: Es gibt nur einen Dalai Lama, der immer wiedergeboren wird, sodass dessen Nachfolge nicht durch Vergleich „würdiger“ Nachfolger reguliert wird, sondern das Problem der Substitution auf das Suchen und Finden der gerade aktuellen Reinkarnation des Dalai Lama verlagert wird. Die Substitution muss dann als Erkenntnis stilisiert werden und darf nicht den Mönchen als Entscheidung zugerechnet werden (was im Übrigen moderne Organisation voraussetzen würde).

${ }^{10}$ Seit der 1971 erfolgten Einführung der Kategorie der Least Developed Countries in der UN haben lediglich vier Staaten den Aufstieg zu Less Developed Countries geschafft (United Nations Conference on Trade and Development 2015, S. iii).
} 
Diese Schematisierung von Konstanz und Variabilität hat also auch Auswirkungen darauf, welche Leistungen von Mitgliedsstaaten erwartet werden können und für welche konstant bleibenden Bedingungen Rücksichtnahme in Anspruch genommen werden kann.

\section{Leistungserwartungen zwischen Anspruch und Rücksichtnahme}

Die Problematik des Austarierens von Rücksichtnahme und Inanspruchnahme wird unter Bedingungen der modernen Gesellschaft häufig unter dem Gesichtspunkt der Dominanz von Leistungsansprüchen gegenüber der Rücksichtnahme auf konstante Qualitäten charakterisiert (siehe als locus classicus: McClelland 1976). Sie lässt sich andernorts als These des Übergangs von ascription zu achievement (Linton 1964) bzw. in überarbeiteter Form im Übergang von quality zu performance (Parsons 1953) finden. Wie hier aber zu zeigen versucht wurde, werden in Rankings zugeschriebene konstante Qualitäten (z.B. in der Form klassifizierender Gattungsbegriffe) mit zeitlich variierenden Leistungsziffern gekoppelt, sodass eher davon ausgegangen werden müsste, dass nicht achievement bzw. performance gegenüber ascription oder quality an Bedeutung gewonnen hätten, sondern dass den jeweiligen Unterscheidungen besondere Bedeutung beigemessen wird. Soziale Systeme lassen sich (jedenfalls unter modernen Bedingungen) offenbar nicht auf eine Seite des Qualität/Leistung-Schemas festlegen, sondern dürften sich vielmehr dahingehend rationalisieren, dass beide Seiten dieses Schemas flexibel eingesetzt und aufeinander bezogen werden, was aber Asymmetrien und unterschiedlich verteilte Beweislasten in beiden (!) Richtungen des Schemas nicht ausschließen muss (Luhmann 1981, S. 87ff.). Sie können aber nicht ausschließlich auf konstante Qualitäten hin getrimmt werden, sodass in IOs etwa China ungeachtet aller ökonomischen Fortschritte weiterhin als Entwicklungsland zu klassifizieren wäre. Andererseits können IOs nicht auf die komplexitätsreduzierende Funktion von zeitlich als konstant anzunehmenden Prämissen verzichten, um Entscheidungen ausschließlich von sich ständig ändernden Umweltleistungen (v.a.: der Mitgliedsstaaten) abhängig zu machen. Auch IOs müssen sowohl Qualitäten als auch Leistungen ihrer Mitgliedsstaaten so behandeln, dass sie wechselseitig füreinander als anerkannte Entscheidungsgesichtspunkte fungieren können und damit die Möglichkeit des „Führungswechsels“ zwischen beiden Aspekten gewahrt bleibt. 
Im zeitlichen Planungshorizont von Reformen muss schließlich auch mit konstanten, weil politisch unentscheidbaren Entscheidungsprämissen gerechnet werden (Luhmann 2000, S. 240ff.), die z.B. als Ausdruck nationaler „Kultur“ sogar affirmiert werden können. Aus dem Vergleich mit konkurrierenden Spitzenreitern von Rankings kann bei „Entwicklungsnachzüglern“ schließlich nicht nur der Eindruck einer relativen Deprivation entstehen, auf den mit Übernahme und Diffusion von Erwartungen reagiert wird, sondern es können auch äquivalent dazu Abgrenzungs- und Distinktionsprozesse einsetzen ${ }^{11}$, bei denen kulturelle Eigenheiten und bewahrenswerte Traditionen positiv bewertet werden können (Holzer 1999, S. 102ff.). Die „Verlierer“ von Klassifikationen können dann durch aktive Aneignung und Uminterpretation von Erwartungen eigenständige Entwicklungspfade einschlagen (ebd., S. 105) oder, um es mit Bourdieu zu formulieren, aus der Not eine Tugend machen und einen amor fati bzw. einen „Notwendigkeitsgeschmack“ kultivieren, der „bewirkt, daß man hat, was man mag, weil man mag, was man hat, nämlich die Eigenschaften und Merkmale, die einem de facto zugeteilt und durch Klassifikation de jure zugewiesen werden“ (Bourdieu 1992, S. 286). Die Mitgliedsstaaten (und das gilt sowohl für Gewinner als auch für Verlierer von Rankings) können aber auch mit Hinweisen auf „Klassenzugehörigkeit“, auf unentscheidbare Entscheidungsprämissen oder auf kulturelle Rücksichtnahmepflichten Konkurrenz durch Distinktion (bzw. analog dazu: Vergleich durch Klassifikation) substituieren und sich damit vor Leistungs- und Reformansprüchen schützen. ${ }^{12}$ Die Beobachtung von Kultur wird dann nicht durch ein Vergleichsinteresse angeleitet (so aber Luhmann 1999), sondern als zu Vergleichen gegenläufiger Mechanismus des Aufbaus von Vergleichsschwellen eingesetzt, mit dem Erwartungsenttäuschungen gesichtsschonend verarbeitet und kognitive Dissonanzen aufgelöst werden können.

Trifft all dies zu, erscheint es nur folgerichtig, dass leistungsneutrale Größen wie etwa Kultur in die Gleichung eingeführt werden, sodass für die Verhandlungen über Zielprogramme in IOs nicht nur zeitlich variable Leistungen, sondern auch

\footnotetext{
11 Die Äquivalenzbeziehung zwischen Konkurrenz und Distinktion sei hier nochmals betont um funktionalistische Kurzschlüsse zu vermeiden, wie sie etwa Sauder und Espeland (2009) im Anschluss an Foucault nahelegen. Dabei gehen sie davon aus, dass die konkurrenzstiftende Wirkung von Rankings einen „Druck“ auslöse, der zur Internalisierung von Erwartungen und zur Disziplinierung führen würde. Diese teleologische Denkfigur suggeriert, dass Knappheitsprobleme (z.B. in der Form knappen Prestiges) durch „Druck“ ganz bestimmte Lösungen mobilisieren.

${ }^{12}$ So konnte etwa in Frankreich der „PISA-Schock“ ausbleiben, weil in der medialen Berichterstattung, die sich insgesamt in überschaubaren Grenzen hielt, der angelsächsische kulturelle biais der Studie kritisiert wurde, mit dem vermeintliche Eigenheiten des französischen Bildungssystems nicht erfasst worden seien (Schmitt 2002).
} 
berücksichtigenswerte, zeitlich als konstant anzunehmende Qualitäten wie eben „kulturelle Identität“ einkalkuliert werden müssen. Gleichwohl kann man sich seit Heraklit vorstellen, dass nichts dem Wandel der Zeit standzuhalten vermag und man wird Ähnliches auch für nationale „Kulturen“ unterstellen können. Der in der Weltbank neuerdings praktizierte Verzicht auf Klassifikationen nach dem Schema developed/developing countries, an deren Stelle die Zusammenfassung von Staaten zu globalen Regionen trat, verdankte sich schließlich der Annahme der Sustainable Development Goals der UN, dass die Welt insgesamt als eine sich entwickelnde Welt (developing world) begriffen werden müsse und daher alle Staaten als Adressaten dieser Entwicklungspolitik (d. h.: als „Entwicklungsländer“) zu verstehen seien (United Nations 2015). Es stellt sich aber die Frage, wie bestimmte Größen (abgesehen von der geographischen Lage eines Mitgliedsstaates) in der longue durée noch konstant gehalten werden können und wie zeitliche Kontingenz dahingehend schematisiert werden kann, dass zwischen konstant bleibenden und ohnehin variablen Planungsprämissen unterschieden werden kann.

\section{Leistungserwartungen zwischen Kurzfristigkeit und Langfristigkeit}

IOs haben es als Meta-Organisationen mit Mitgliedern zu tun, deren Lebenserwartung in der Regel nicht seriös angegeben werden kann (Ahrne und Brunsson 2008, S. 58ff.). Auf welche unreformierbaren konstanten Qualitäten sollte eine IO gegenüber ihren unsterblichen Mitgliedsstaaten angesichts dieser langfristigen Zeitperspektive Rücksicht nehmen, um sie nicht mit unrealistischen und enttäuschungsanfälligen Leistungserwartungen $\mathrm{zu}$ konfrontieren? Es steht $\mathrm{zu}$ vermuten, dass hier Fristen ein funktionales Äquivalent für den Staatskollaps darstellen und viel davon abhängen wird, welche Fristen für die Umsetzung von Leistungs- und Reformforderungen konzediert werden.

Fristen haben zum einen eine wichtige Koordinationsfunktion für Entscheidungsrelais, die es ermöglichen, „die Vorteile von gleichzeitiger Parallelarbeit mit linearen Strukturen zu verbinden. Sie sind besonders wichtig, wenn eine regionale oder sonstwie segmentäre Differenzierung vorliegt und zentral verwaltet werden muss" (Luhmann 2000, S. 175). Demnach muss es nicht überraschen, dass in der zeitlichen Koordination solcher Entscheidungszusammenhänge ein Hauptaufgabengebiet und zugleich ein Hauptquell zeitlicher Autonomie von IOs liegt (Ekengren 2002). Befristete Zielsetzungen 
erzeugen aber auch in Anbetracht einer offenen Zukunft Selektionszwänge und stutzen programmförmig formulierte Leistungsanforderungen auf kleinere Formate herunter: Je kürzer die Frist, desto weniger kann an Reformen geleistet werden. Es können Zweckprogramme eingerichtet werden, die durch Befristung zeitlich dermaßen spezifiziert sind, dass sie streng genommen nur einmal durchgeführt werden können und eher Projektcharakter annehmen (Luhmann 2000, S. 272) - Beispiele wie die Millenium Development Goals der UN oder die Lissabon-Strategie der EU, bis 2010 zum dynamischsten Wirtschaftsraum der Welt zu werden, ließen sich hier anführen. In diesen Projekten kann Zeit auch bei großzügigeren Fristen insofern wieder verknappt werden, als Reformleistungen in regelmäßigen Abständen mit Rankings zwischenevaluiert werden und Mitgliedsstaaten nun dazu angehalten werden, z.B. im Jahresrhythmus sichtbare Ergebnisse vorzuweisen. Das ermöglicht eine Differenzierung zweier Zeitperspektiven, die einander gegenseitig stützen können und sich wechselseitig in Erinnerung bringen: Die langfristige Perspektive des Projektendes kommt nicht erst kurz vor Fristablauf zum Tragen, sondern kann sich bereits bei früheren Zwischenevaluationen bemerkbar machen. Andererseits kann man auch zeitaufwändigere Reformen wagen, deren Resultate sich nicht bei jeder Zwischenevaluation positiv niederschlagen müssen und deren Effekte relativ spät messbar werden.

Bei dieser Differenzierung von Zeitperspektiven können IOs auch jenen Effekt von Fristsetzungen nutzen, der in der Verschiebung der Aufmerksamkeit auf zeitlich befristete Entscheidungsmaterien liegt (Luhmann 1971a). Je knapper Zeit im Hinblick auf den Fristablauf von Projekten in Mitgliedsstaaten erlebt wird, desto eher steigt die Chance, dass diesen Projekten auch Aufmerksamkeit zuteilwird. Auch dieser Effekt lässt sich durch regelmäßige Zwischenevaluationen und Leistungserhebungen steigern, sodass bereits vor Ablauf des Projekts Vorsprünge und Rückstände beobachtbar werden, die in den Rankings in der Form von Auf- und Abstiegen symbolisiert werden. Dieses Arrangement ermöglicht nicht zuletzt auch die Beobachtung von zeitlichen Positionsschwankungen, die im Kontext von IOs auch als Statusänderungen oder „Karrieren“ interpretiert werden können. Der folgende Abschnitt widmet sich daher möglichen Folgen von Rankings für die organisatorische Zuordnung von Kompetenzen bzw. Status und deren Koordination mit anderen Entscheidungsprämissen in IOs. 


\section{Rankings und Kommunikationswege in IOs}

Rankings können, wie oben gezeigt wurde, in gewissen Hinsichten Mitgliedsstaaten und Programme derart aneinanderkoppeln, dass beide Entscheidungsprämissen nicht unabhängig voneinander variieren und wechselseitig als Entscheidungsgesichtspunkte gewählt werden können. Aber wie können Rankings die beiden genannten Entscheidungsprämissen mit der dritten wichtigen Entscheidungsprämisse, den Kommunikationswegen, verknüpfen und gleichzeitig füreinander flexibel halten?

Üblicherweise kann man in klassischen Organisationen an der Stellenordnung ablesen, auf welche Mitglieder Kompetenzen verteilt sind und welche Kommunikationswege für bestimmte Entscheidungsprogramme einzuhalten sind, d. h. welche kompetenten Stellen im Entscheidungsprozess $\mathrm{zu}$ berücksichtigen sind. Man möchte in einer Universität eine Reisekostenabrechnung einreichen, wirft einen Blick auf die Stellenordnung (z.B. in Form eines Organigramms) und wendet sich im Idealfall an die entsprechende Stelle im Rechnungswesen und nicht etwa an den Rektor. Wie man an diesem Beispiel sehen kann, vermittelt eine Stellenordnung daher zugleich auch Kommunikationswege, die in klassischen Organisationen an Formen der Arbeitsteilung bzw. der hierarchischen und funktionalen Differenzierung gekoppelt sind (Luhmann 1995, S. 144f.).

In IOs hingegen werden die Kommunikationswege zwischen Mitgliedsstaaten insbesondere durch die primär segmentäre Differenzierungsform des weltpolitischen Systems in funktional gleichartige Subsysteme, nämlich Nationalstaaten, konditioniert. Die formalisierten Kommunikationswege zwischen Mitgliedsstaaten verlaufen daher typischerweise nicht entlang der funktionalen Differenzierung in Politikbereiche oder in der Form hierarchisch differenzierter Weisungskompetenzen. Stattdessen werden in IOs entweder alle Mitgliedsstaaten gleichermaßen als Mitglieder der Organisation bzw. eines Entscheidungsgremiums adressiert (z.B. die UN-Vollversammlung, der Rat der EU etc.) oder es werden konkrete Mitgliedsstaaten namentlich als Ansprechpartner genannt (z.B. als ständige Mitglieder des UN-Sicherheitsrats). ${ }^{13}$ Auf diese Weise drückt sich in IOs die formale Egalität der Mitgliedsstaaten aus, die zunächst, einfach formuliert, zu wenig Differenzen erzeugt, an denen sich die Strukturierung von Kommunikationswegen

\footnotetext{
13 Ausnahmen gelten für die Adressierung von Mitgliedsstaaten in Vorsitzfunktionen. An der zeitlichen Befristung und dem häufig anzutreffenden Rotationsprinzip solcher Vorsitzfunktionen kann man auch die Prekarität solcher „Stellenvergaben“ ablesen.
} 
zwischen Mitgliedsstaaten orientieren könnte. Allerdings schließt diese Egalität nicht weitere, organisationsinterne Differenzierungen aus, im Gegenteil: Das Gleichheitssymbol formaler Egalität „,souveräner“ Mitgliedsstaaten hat nur die Funktion, die unvermeidbaren organisationsinternen Ungleichheiten von ebenso unvermeidbaren organisationsexternen Ungleichheiten $\mathrm{zu}$ isolieren. Es ist damit ein Ausdruck der Autonomie und Ausdifferenzierung des Systems (allgemein hierzu Kieserling 2006). Zu den organisationsinternen Ungleichheiten gehören etwa differenzierbare Stimm- und Vetorechte, sodass es sich in der Praxis empfiehlt, bestimmte Staaten entsprechend früh in Meinungsbildungs- und Entscheidungsprozesse einzubinden. Aber diese Formen der Allokation von Kompetenz und Status sind gegenüber variierenden Politikprogrammen üblicherweise indifferent, sodass sie kaum zur Kopplung von Kommunikationswegen und Programmen beitragen. Trotz sachlich variierender Programme müssen in IOs also meistens annähernd gleichbleibende formalisierte Kommunikationswege zwischen Mitgliedsstaaten berücksichtigt werden. Zwar gibt es häufig unterschiedliche Gremien und Ausschüsse, die mit bestimmten Politikbereichen befasst sind. Jedoch sind in diesen Gremien üblicherweise alle Mitgliedsstaaten vertreten, die dadurch gleichermaßen an Entscheidungsprozessen beteiligt sind.

Es gibt also kaum ausgeprägte Kompetenzarchitekturen, an welchen sich eine Formalisierung von Kommunikationswegen orientieren könnte und die konkrete Staaten als Ansprechpartner für spezielle Politikprogramme vorsehen würden. Es gibt keine Vorschriften, die besagen, dass man sich in Fragen der Hochseefischerei an Russland und in Fragen der Bildungspolitik an die USA zu wenden habe. Die Programme in IOs, die sich aber auf Politikbereiche wie Bildungspolitik, Wirtschaftspolitik, Wissenschaftspolitik etc. beziehen und entlang der funktionalen Differenzierung der Gesellschaft rationalisiert werden, müssen dennoch durch weitgehend invariable formalisierte Kommunikationswege zwischen Mitgliedsstaaten prozessiert werden, die sich an der segmentären Differenzierung der Weltpolitik orientieren.

Diese Problemexploration führt also auf die Kernfrage zurück, wie in IOs Entscheidungsprämissen füreinander variabel gehalten werden können, sodass nicht immer Programme an konstant bleibende Mitglieder und Kommunikationswege angepasst werden müssen. Eine Lösung scheint darin zu liegen, wenn schon nicht Stellen, so zumindest bestimmte „Rollen“ $\mathrm{zu}$ institutionalisieren, welche die Aufmerksamkeit je nach Programm auf auswechselbare Rollenträger bzw. 
Mitgliedsstaaten lenken und aus Sicht des Programms „richtige“ Kommunikationswege zwischen Mitgliedsstaaten nahelegen. Das setzt aber voraus, dass trotz formaler Egalität der Mitgliedsstaaten bestimmte sachlich begründbare Statusungleichheiten erzeugt, verändert und von den Mitgliedsstaaten akzeptiert werden können. Diese Funktion, die in klassischen Organisationen Stellenordnungen erfüllen, kann in IOs von Rankings bearbeitet werden. Sie integrieren dafür zwei Mechanismen: Erstens verlagern sie das Problem der Statusdifferenzierung in die Sphäre der Informalität, um von dort aus im Sinne organisationsinterner Prämissen Kommunikationswege zu strukturieren und die Imitation erfolgreicher Vorbilder $\mathrm{zu}$ motivieren. Zweitens erfordern Rankings Entscheidungsverfahren, in deren Verlauf sich die Mitgliedsstaaten mit Rankings und ihren positiven und negativen Ergebnissen identifizieren können. Beide Mechanismen sollen in den folgenden Abschnitten näher charakterisiert werden.

\section{Rankings und die „Formalisierung“ informeller Kommunikationswege}

Die Kopplung von Mitgliedsstaaten, Programmen und Kommunikationswegen kann unter der Bedingung gelingen, dass unter organisationsinternen Leistungsgesichtspunkten je nach Programm bzw. Politikbereich die Aufmerksamkeit auf bestimmte Mitgliedsstaaten fokussiert werden kann, die auch in der Lage sind, eigene Situationsdefinitionen durchzusetzen. Es geht also, anders formuliert, um die Verteilung von Rang und Status nach organisationsinternen Prämissen (vgl. Luhmann 1995, S. 159f.). Was im Normalfall die Ausstattung einer Stelle mit Weisungskompetenzen und Zeichnungspflichten bewirkt, die zumindest den formalisierten Status der Stelleninhaber begründet, muss für Mitgliedsstaaten in IOs durch äquivalente Problemlösungen geleistet werden. Hier können Rankings für IOs wiederum Abhilfe leisten, indem sie die Frage nach sachlicher Kompetenz dem Muskelspiel der Mitgliedsstaaten entziehen und eine organisationsinterne Kompetenzordnung simulieren, in der Status sachlich je nach Programm, aber auch zeitlich mit den Positionsschwankungen in Rankings variiert. Rankings lassen dafür Kompetenz als Gegenstand eines organisationsinternen Nullsummenspiels ${ }^{14}$ erscheinen

\footnotetext{
14 Eine auffallende Parallele dazu bilden demokratische Wahlverfahren, in denen Macht in der Form quantifizierbarer Stimmen einerseits und begrenzter Stellen (bzw. Ämter) andererseits unter der Prämisse der Nullsummenkonstanz beobachtbar werden und dabei eine Machtordnung etabliert wird, die sich nicht mehr unmittelbar aus den ansonsten verfügbaren Ressourcen politischer Konkurrenten ableiten lässt (Luhmann 1983, S. 176f.).
} 
(Werron 2012, S. 178), in welchem Staaten nur auf Kosten anderer Staaten besondere sachliche Kompetenzen z.B. in Fragen der Bildungs- oder Umweltpolitik nachweisen können, sodass Rankings eine Orientierungsfunktion übernehmen, die für gewöhnlich Stellenordnungen erfüllen. Auf diese Weise kann die Aufmerksamkeit auf bestimmte Staaten gelenkt werden, wenn in politischen peer review-Prozessen nach kompetenten Ansprechpartnern für bestimmte Politikprogramme gefragt wird, oder nach Vorbildern, deren best practices imitiert werden können (Knill und Lenschow 2005, S. 120; Nedergaard 2006). Die Strukturierung von Kommunikationswegen beruht nicht auf einer formalisierten Kompetenzordnung, sondern setzt auf einer informellen Ebene an, wodurch die offizielle Selbstdarstellung der Mitgliedsstaaten und deren formale Gleichheit gewahrt bleiben können. Anstelle von Weisungsbefugnissen gewinnen informelle Formen des Einflusses in Formen von Reputation und Führung bzw. sachlich und sozial generalisiertem Einfluss an Bedeutung (Luhmann 2012, S. 84f.).

Der Erfolg dieser Art von Einfluss hängt zum einen davon ab, dass Mitgliedsstaaten mit hoher Reputation Gründe für die Richtigkeit ihrer Entscheidungen angeben können, die relativ unkritisch angenommen werden und eine Weisungskompetenz substituieren können. Zum anderen setzt er voraus, dass die Bereitschaft von Mitgliedsstaaten, bestimmte führende Staaten und deren nationale Politiken nachzuahmen, durch die Vermutung verstärkt wird, dass auch andere diesen einflussreichen Vorbildern folgen werden. In IOs können beide Einflussformen zu informellen Erwartungssets gerinnen, die man auch als Leadership bezeichnen könnte. Welcher Mitgliedsstaat in welchen Politikbereichen solche Leadership-Funktionen einnehmen und eigene Problemlösungen als Beispiele für best practice darstellen kann, hängt von der Verteilung von Selbstdarstellungschancen sowie von der Möglichkeit einzelner Mitgliedsstaaten $\mathrm{ab}$, ihren Einfluss geltend $\mathrm{zu}$ machen und eigene Problem- und Situationsdefinitionen durchzusetzen. Gelingt es einer IO, die im Sinne ihrer Entscheidungsprogramme „richtigen“ Staaten als leader einzusetzen, können Programme und Kommunikationswege auch auf diese Weise miteinander gekoppelt werden. ${ }^{15}$

Das durch Rankings generierte symbolische Kapital kann hier durchaus eine Rolle spielen. Gleichwohl speisen sich die Möglichkeiten der Einflussnahme von Staaten in IOs

\footnotetext{
15 Wobei die Diffusion von best practices im Sinne der IO nicht zwingend Kommunikation zwischen den Mitgliedsstaaten erfordern muss.
} 
nicht allein aus Rankings. Es wird vielmehr damit zu rechnen sein, dass man sich bei der Wahl von Kooperationspartnern und Vorbildern an allen möglichen und nicht immer an sachlich begründbaren informellen Gesichtspunkten orientieren wird, was die Effektivität von Rankings in dieser Hinsicht stark relativieren dürfte (Eberlein 2003; Mak 2003). Die Problematik der Verteilung von Status und Einflusschancen für bestimmte Sachbereiche verlagert sich eher auf die Sozialisation von ständigen diplomatischen Vertretern der Mitgliedsstaaten in der informellen Hackordnung der diplomatic community (Pouliot 2011). Die Erfolgsaussichten von Versuchen, diese informellen Kommunikationswege durch Rankings zu „formalisieren“, sind also denkbar gering, und sie hängen vor allem davon ab, dass die Ergebnisse von Rankings zu einem Element der Selbstdarstellung von Mitgliedsstaaten werden. Diese sind aber in ihren Selbstdarstellungen meistens schon festgelegt, sodass IOs mit anderen Mitteln jene „goldenen Brücken“ (Luhmann 1995, S. 147) bauen müssen, die es den Mitgliedsstaaten ermöglichen, ihre festgelegten Selbstdarstellungen zu ändern. Mit der Verlagerung in Zonen der Informalität wurde bereits eine Brücke genannt. Eine weitere Brücke bietet die Einbindung in jene Verfahren, die der Erstellung von Rankings vorausgehen und im folgenden Abschnitt zu skizzieren sein werden.

\section{Rankings zwischen Statuserkenntnis und -entscheidung in IOs}

Für Staaten kann es attraktiv sein, in möglichst vielen Politikbereichen Einfluss zu steigern, indem sie leader werden und in dieser Funktion möglichst nicht ersetzt werden. Dabei versteht es sich von selbst, dass um Einfluss konkurrierende Staaten sich nicht zwangsläufig an Leistungsgesichtspunkten von IOs orientieren. Für IOs hingegen kommt es primär darauf an, die Verteilung von Rang, Status und Aufmerksamkeit dem freien Selbstdarstellungsmarkt der Mitgliedsstaaten $\mathrm{zu}$ entziehen und systeminterne Verteilungsmechanismen so zu institutionalisieren, dass die Träger solcher LeadershipFunktionen für Organisationszwecke substituiert werden können. Außerdem müssen IOs in der Lage sein, die Besetzung dieser Rollen nach organisationsinternen Leistungsgesichtspunkten an veränderte Umweltlagen anzupassen, ohne durch die Umwelt (z.B. den systemexternen Status der Mitgliedsstaaten) determiniert zu werden. Die Verteilung von Status in IOs muss also in Form von Entscheidungen prozessiert werden. Es macht nämlich „einen wesentlichen Unterschied, ob die Statusbegründung sozial als Erkenntnis oder als Entscheidung institutionalisiert ist" (Luhmann 1995, S. 
167). Nur im letzteren Fall kann die Organisation als Beteiligte am Status (und der „Karriere“) eines Mitgliedsstaates beobachtet werden und eine organisationsinterne Statusordnung institutionalisieren, die weitgehend unabhängig von der Umwelt bestehen und variieren kann. Aber gerade die Ergebnisse (v.a. quantifizierender) Rankings werden primär als Erleben und nicht als Handeln, als objektive Erkenntnisse wissenschaftlicher Methoden und weniger als Entscheidungen in politischen Verfahren stilisiert (Heintz 2007, 2010).

Auf den ersten Blick scheinen IOs daher genau das Gegenteil von dem zu erreichen, was eigentlich bewirkt werden soll: Rankings würden demnach die in der Umwelt von IOs ohnehin vorhandenen Statusungleichheiten zwischen den Mitgliedsstaaten lediglich abbilden und davon eine interne „Kopie“ anfertigen, die von IOs nicht mehr für eigene Zwecke manipulierbar wäre. Die Mitgliedsstaaten müssten dann ihr organisationsexternes symbolisches Kapital nicht erst in der Organisation konvertieren, sondern könnten beinahe so miteinander kommunizieren, wie sie es auch außerhalb von IOs täten. Aber diese Annahme übersieht, dass Rankingergebnisse allein noch keine unmittelbaren, automatischen Auswirkungen auf den Status von Mitgliedsstaaten haben, sondern Statusänderungen erst in Entscheidungen konvertiert werden müssen. So erfordert z.B. in der UNO der Aufstieg eines Mitgliedsstaates zu einem less developed country einen politischen Beschluss der Mitgliedsstaaten - sei es wegen oder gerade trotz bestimmter Ranking-Ergebnisse. ${ }^{16}$ Außerdem muss über die Durchführung von Rankings sowie über deren Leistungsziele, Messverfahren und Indikatoren entschieden werden (Merry 2014). Solche politischen Entscheidungen können die Ergebnisse von Rankings erheblich beeinflussen, wodurch für die Statusbegründung von Mitgliedsstaaten wiederum organisationsinterne Entscheidungen und „objektive“ Erkenntnisse miteinander gekoppelt werden. ${ }^{17} \mathrm{Je}$ mehr die Mitgliedsstaaten über Details von Ranking-Verfahren mitbestimmen können, desto eher können sie sich dann aber auch mit dem Verfahren identifizieren. Die IO kann dadurch nicht nur tertium

\footnotetext{
16 Siehe z.B. die UN-Resolution zu Angola (United Nations 2016). Auch hieran sieht man, wie prekär die Autonomie von IOs trotz Rankings bleiben kann.

${ }^{17}$ Diese Kopplung zwischen Entscheidung und Erkenntnis birgt freilich die Gefahr, dass sich Mitgliedsstaaten für Indikatoren einsetzen, von denen sie bessere Rankingergebnisse erwarten können. Aber diese Möglichkeit wird zum einen dadurch beschränkt, dass man die Ergebnisse eines Rankings kaum im Voraus kalkulieren kann, da die dafür nötigen Daten erst erhoben werden müssen. Zum anderen werden Rankingverfahren im Regelfall von eigenen Abteilungen in IOs durchgeführt, sodass die Entscheidung über möglicherweise entscheidende statistische Feinheiten deren wissenschaftlicher Expertise überlassen bleiben muss.
} 
comparationis, sondern auch tertius gaudens sein: Je eher sich die Mitgliedsstaaten durch ihre Mitwirkung an diesen Verfahren auch in ihren Selbstdarstellungen auf ein Ranking einlassen, desto unweigerlicher werden die Ergebnisse, so unangenehm diese später auch ausfallen mögen, zum Element ihrer Selbstdarstellung: Teilnahme verpflichtet (Luhmann 1983, S. 91ff.; 187). Wer über das Ranking-Prozedere mitentschieden hat und sich danach über die Ergebnisse beklagt, stünde allenfalls als schlechter Verlierer da. Es empfiehlt sich daher meistens, sich auch mit schlechten Rankingergebnissen abzufinden, zumal sich immer irgendein Gesichtspunkt finden lässt, von dem aus die eigene Situation akzeptabler erscheint, als ein Ranking vielleicht nahelegen mag.

Wenn sich die Mitgliedsstaaten unter diesen Bedingungen an den organisationsinternen Zielen und Indikatoren orientieren und auch bei eigenem schlechten Abschneiden die Führungsfunktion anderer Staaten akzeptieren, kann die IO ihre Unabhängigkeit von systemexternen Bewertungskriterien ihrer Mitgliedsstaaten steigern und durch Rankings nicht nur „Karrieren“, sondern auch je nach Politikprogramm variierende Kompetenz- und Statusordnungen simulieren, ohne dafür eine Stellenstruktur einrichten zu müssen. Treffen diese Annahmen zu, scheint auch hier die These einer Äquivalenz zwischen einer Stellenstruktur und Leistungsrankings eine gewisse Tragfähigkeit zu haben.

\section{Fazit}

Die hier angestellten Überlegungen hatten zum Ziel, eine organisationssoziologische Perspektive auf leistungsvergleichende Rankings in IOs zu entwickeln. Als Ergänzung zu der weitgehend unbestrittenen These, dass mit solchen Rankings Probleme der global governance bearbeitet werden, indem Staaten durch konkurrenzstiftende Leistungsvergleiche zur compliance motiviert werden, wurde hier die These erprobt, dass Rankings anstelle einer Stellenordnung die Funktion der Koordination und Kontrolle von Entscheidungsprämissen erfüllen können. Entscheidungsprämissen werden durch Rankings so aneinander gekoppelt, dass sie nicht unabhängig voneinander variieren, wodurch sie wechselseitig füreinander als Substitutionsgesichtspunkte fungieren können. Es wurde darüber hinaus versucht zu zeigen, dass IOs mit Hilfe von Rankings ihre Mitgliedsstaaten in gewissen Hinsichten als substituierbar behandeln können. Dies erfolgt bspw. durch die Einteilung in 
Leistungsgruppen bzw. durch Subsumption unter entwicklungspolitische Gattungsbegriffe, mit denen sehr unterschiedliche Staaten als substituierbare Anwendungsfälle spezifischer Programme beobachtet werden können. Aber auch Kommunikationswege können mit Programmen und Mitgliedsstaaten gekoppelt werden, indem informelle Leadership-Rollen in bestimmten Sachbereichen mit wechselnden nationalstaatlichen Ranking-Spitzenreitern besetzt werden können. IOs können mit solchen Mitteln interne Redundanzen steigern, indem kontingente, substituierbare Entscheidungsprämissen auf nicht-kontingente Weise miteinander verknüpft werden, was die Entstehung von organisatorischen Routinen erleichtert, die auf variierende Umweltinformationen und Leistungen von Mitgliedsstaaten ähnliche Systemreaktionen folgen lassen können. Gleichzeitig kann aber auch die Variabilität und Flexibilität von IOs gesteigert werden, da durch die Umweltinformationen, die durch leistungsvergleichende Rankings generiert werden, Programme kontrolliert und ggfs. an neue Umweltlagen angepasst werden können.

Ein weiterer Befund der funktionalen Analyse von Rankings war, dass sie nicht nur Vergleiche, sondern auch Klassifikationen erzeugen, mit denen Substitutionsschwellen aufgebaut werden. Dadurch erscheinen die Staaten lediglich innerhalb bestimmter Klassen als vergleichbar, sodass Leistungskonkurrenz teilweise durch Distinktion substituiert werden kann. Darüber hinaus bewirken schlechte Rankingergebnisse nicht automatisch erhöhte Anstrengungen und Leistungen. Stattdessen können leistungsneutrale Faktoren wie z.B. „Kultur“ geltend gemacht werden, mit denen enttäuschte Leistungserwartungen gesichtsschonend im Modus kognitiver Dissonanzauflösung verarbeitet werden können, und dadurch weitgehend folgenlos bleiben.

Die Funktion von Rankings kann also nicht allein aus der Leistungskonkurrenz zwischen Staaten abgeleitet werden. Dennoch orientieren sich die wissenschaftlichen Einschätzungen über die praktischen Effekte von internationalen Rankings an dieser manifesten Funktion und kommen meistens zu pessimistischen Befunden. Rankings seien etwa nur bedingt effektive Steuerungsinstrumente, da die Spitzenreiter von Rankings Statistiken frisierten bzw. window dressing betrieben und sich auf ihren Lorbeeren ausruhten, während die Nachzügler trotz aller Reformbemühungen durch schlechte Rankingergebnisse demotiviert würden (van der Vleuten und Verloo 2012). Darüber hinaus werden Erhebungsmethoden und Indikatorenauswahl (z.B. Ravallion 
2012) problematisiert. Beklagt wird auch, dass die Veröffentlichung von Rankings dazu führten, dass mitunter eher Aspekte der „Performanz" und weniger performance im Vordergrund stünden (Espeland und Sauder 2007; Nullmeier 2004). ${ }^{18}$ Diese Diagnosen haben zweifellos ihre Berechtigung. Die hier durchgeführte funktionale Analyse hatte im Gegensatz dazu eine eher latente Funktion im Kontext von IOs im Fokus und führt daher auch zu anderen Ergebnissen. Sie konnte zunächst Potentiale und Möglichkeiten von Rankings für die Autonomie von IOs aufzeigen, die aber in der Praxis variierende Bedingungen vorfinden, und sich in stark integrierten Organisationen wie der EU anders ausprägen als in einer Organisation wie der OECD. Das berührt aber nicht die funktionale Analyse und die These der Äquivalenz zwischen Rankings und Stellenordnungen. Die Angabe einer Funktion hat schließlich nur die Funktion, den Vergleich kontingenter Problemlösungen zu ermöglichen. Davon zu trennen sind aber Aussagen über den Erfolg oder die Wahrscheinlichkeit bestimmter Problemlösungen in ihrem praktischen Vollzug. Ob sich Menschen nur durch Opiumkonsum oder nur durch Religion in Rauschzustände versetzen, ändert nichts an der Äquivalenz beider Lösungen. Im Sinne theoretischer Generalisierung wurden hier daher Problemzusammenhänge und Bedingungen der Möglichkeit des „faktischen“ Funktionierens von Rankings herausgearbeitet. $\mathrm{Zu}$ den wichtigsten Bedingungen dürfte zählen, inwiefern es IOs gelingt, Konditionalprogramme und Routinen auszubilden, die unabhängig vom Einfluss der Mitgliedsstaaten konstant gehalten werden können. Dies setzt generalisierte Programme voraus, die für alle Mitgliedsstaaten gleichermaßen gelten und ohne Konsultation der Mitgliedsstaaten angewendet werden können. Aber solange die wichtigsten Entscheidungen in IOs (vor allem: Entscheidungen über Entscheidungsprämissen) im Modus diplomatischer Verhandlungen getroffen werden, dürfte damit zu rechnen sein, dass Mitgliedsstaaten Ausnahmen und „individuelle“ Lösungen für sich reklamieren werden, die der Generalisierbarkeit von Programmen entgegenstehen und den Entscheidungsspielräumen in IOs enge Grenzen setzen. Wie IOs unter diesen Bedingungen Konstanz und Flexibilität, Offenheit und Geschlossenheit,

\footnotetext{
${ }^{18}$ In diesem Zusammenhang sei erwähnt, dass performance im Englischen ja zunächst die rituelle Erfüllung einer Rechtspflicht bedeutete (wie auch Leistung im Deutschen eher im Kontext von Vergleichen, d. h. von Verträgen gebraucht wurde). Angesichts ungewisser Zukunftsperspektiven und eines noch geringen Systemvertrauens in geldbasierte Wirtschaft im England des 16. Jahrhunderts erhielt das Wort performance allerdings die Konnotation der Verstellung, die als Mittel der Verheimlichung eigener Interessen empfohlen wurde (Agnew 1986, S. 82f.).
} 
Abhängigkeit und Unabhängigkeit dann aneinander steigern können, wird wohl weiterhin ein Problem bleiben, bei dem gegenwärtig fraglich bleibt, ob leistungsvergleichende Rankings künftig zu dessen Lösung beitragen können.

\section{Literaturverzeichnis}

Agnew, J.-C. (1986). Worlds apart. The market and the theater in Anglo-American thought, 1550-1750. Cambridge: Cambridge University Press.

Ahrne, G. \& Brunsson, N. (2005). Organizations and Meta-Organizations. Scandinavian Journal of Management 21 (4), 429-449.

Ahrne, G. \& Brunsson, N. (2008). Meta-organizations. Cheltenham: Edward Elgar.

Ahrne, G. \& Brunsson, N. (2012). How Much do Meta-Organizations Affect Their Members? In M. Koch (Hrsg.), Weltorganisationen (S. 57-70). Wiesbaden: VS Verlag für Sozialwissenschaften.

Bourdieu, P. (1992). Die feinen Unterschiede. Kritik der gesellschaftlichen Urteilskraft. Frankfurt a.M.: Suhrkamp.

Bourdieu, P. (2004). Praktische Vernunft. Zur Theorie des Handelns. Frankfurt a.M.: Suhrkamp.

Brunsson, N. \& Jacobsson, B. (Hrsg.). (2002). A world of standards. Oxford: Oxford University Press.

Clark, D. A. (2008). The Capability Approach: Its Development, Critiques and Recent Advances. In R. Ghosh, K. R. Gupta \& P. Maiti (Hrsg.), Development studies (S. 105127). New Delhi: Atlantic Publishers \& Distributors.

Davis, K. E., Fisher, A., Kingsbury, B. \& Merry, S. E. (Hrsg.). (2012). Governance by indicators. Global power through quantification and rankings. Oxford: Oxford University Press.

Drori, G. S., Meyer, J. W. \& Hwang, H. (Hrsg.). (2006). Globalization and organization. World society and organizational change. Oxford: Oxford University Press.

Eberlein, B. (2003). Formal and informal governance in Single Market regulation. In T. Christiansen \& S. Piattoni (Hrsg.), Informal governance in the European Union (S. 150172). Cheltenham: Edward Elgar.

Ekengren, M. (2002). The time of European governance. Manchester: Manchester University Press.

Espeland, W. N. \& Sauder, M. (2007). Rankings and Reactivity: How Public Measures Recreate Social Worlds. American Journal of Sociology 113 (1), 1-40.

Etzioni, A. (1962). International Prestige, Competition and Peaceful Coexistence. European Journal of Sociology 3 (1), 21-41.

Europäische Kommission. (2015). Erweiterungsstrategie der EU (COM(2015) 611), Brüssel. http://ec.europa.eu/enlargement/pdf/key_documents/2015/20151110_ strategy_paper_de.pdf. Zugegriffen 22.02.2016.

Friman, H. R. (2010). Numbers and Certification: Assessing Foreign Compliance in Combating Narcotics and Human Trafficking. In P. Andreas \& K. M. Greenhill (Hrsg.), Sex, drugs, and body counts. The politics of numbers in global crime and conflict (S. 75109). Ithaca, N.Y.: Cornell University Press.

Halliday, T. C. (2012). Legal Yardsticks: International Financial Institutions as Diagnosticians and Designers of the Laws of Nations. In K. E. Davis, A. Fisher, B. Kingsbury \& S. E. Merry (Hrsg.), Governance by indicators. Global power through quantification and rankings (S. 180-216). Oxford: Oxford University Press. 
Heintz, B. (2007). Zahlen, Wissen, Objektivität: Wissenschaftssoziologische Perspektiven. In A. Mennicken \& H. Vollmer (Hrsg.), Zahlenwerk. Kalkulation, Organisation und Gesellschaft (S. 65-85). Wiesbaden: VS Verlag für Sozialwissenschaften.

Heintz, B. (2010). Numerische Differenz. Überlegungen zu einer Soziologie des (quantitativen) Vergleichs. Zeitschrift für Soziologie 39 (3), 162-181.

Heintz, B. (2012). Welterzeugung durch Zahlen. Modelle politischer Differenzierung in internationalen Statistiken, 1948-2010. Soziale Systeme 18 (1+2), 7-39.

Herder, J. G. v. (1802). Ideen zur Philosophie der Geschichte der Menschheit. Dritter Theil. Wien: Anton Pichler.

Holzer, B. (1999). Die Fabrikation von Wundern. Wiesbaden: VS Verlag für Sozialwissenschaften.

Kette, S. \& Tacke, V. (2014). University Rankings. Between Organization and Society. In B. Holzer, F. Kastner \& T. Werron (Hrsg.), From Globalization to World Society. NeoInstitutional and Systems-Theoretical Perspectives (S. 215-236). London, New York: Routledge.

Kieserling, A. (2006). Sinn und Gleichheit. Zur Wissenssoziologie eines modernen Symbols. build, Das Architektenmagazin 6 (6), 21-23.

Knill, C. \& Lenschow, A. (2005). Compliance, communication and competition: patterns of EU environmental policy making and their impact on policy convergence. European Environment 15 (2), 114-128.

Kok, W. (2004). Facing the challenge. The Lisbon strategy for growth and employment: Report from the High level Group chaired by Wim Kok: November 2004. Luxembourg: EUR-OP.

Kühl, S. (2014). The Diffusion of Organizations. The Role of Foreign Aid. In B. Holzer, F. Kastner \& T. Werron (Hrsg.), From Globalization to World Society. Neo-Institutional and Systems-Theoretical Perspectives (S. 258-278). London, New York: Routledge.

Linton, R. (1964). The Study of Man. New York: Appleton-Century-Crofts.

Luhmann, N. (o.J.). Zeithorizonte und Möglichkeitsvorstellungen der Planung, Unveröffentlichtes Manuskript.

Luhmann, N. (1971a). Die Knappheit der Zeit und die Vordringlichkeit des Befristeten. In Politische Planung;. Aufsätze zur Soziologie von Politik und Verwaltung (S. 143-164). Opladen: Westdeutscher Verlag.

Luhmann, N. (1971b). Lob der Routine. In Politische Planung. Aufsätze zur Soziologie von Politik und Verwaltung (S. 113-142). Opladen: Westdeutscher Verlag.

Luhmann, N. (1974a). Funktionale Methode und Systemtheorie. In Soziologische Aufklärung 1. Aufsätze zur Theorie sozialer Systeme (S. 31-53). Opladen: Westdeutscher Verlag

Luhmann, N. (1974b). Soziologie des politischen Systems. In Soziologische Aufklärung 1. Aufsätze zur Theorie sozialer Systeme (S. 154-177). Opladen: Westdeutscher Verlag

Luhmann, N. (1981). Schematismen der Interaktion. In Soziologische Aufklärung 3. Soziales System, Gesellschaft, Organisation (S. 81-100). Opladen: Westdeutscher Verlag

Luhmann, N. (1983). Legitimation durch Verfahren. Frankfurt a.M.: Suhrkamp.

Luhmann, N. (1992a). Die Wissenschaft der Gesellschaft. Frankfurt a.M.: Suhrkamp.

Luhmann, N. (1992b). Status quo als Argument. In A. Kieserling (Hrsg.), Universität als Milieu (S. 16-29). Bielefeld: Haux.

Luhmann, N. (1995). Funktionen und Folgen formaler Organisation. Berlin: Duncker \& Humblot. 
Luhmann, N. (1999). Kultur als historischer Begriff. In Gesellschaftsstruktur und Semantik. Studien zur Wissenssoziologie der modernen Gesellschaft (S. 31-54). Frankfurt a.M.: Suhrkamp.

Luhmann, N. (2000). Organisation und Entscheidung. Opladen: Westdeutscher Verlag Luhmann, N. (2012). Macht. Konstanz: UVK.

Luhmann, N. (2015). Ebenen der Systembildung - Ebenendifferenzierung. In B. Heintz \& H. Tyrell (Hrsg.), Interaktion - Organisation - Gesellschaft revisited. Anwendungen, Erweiterungen, Alternativen (S. 6-39). Stuttgart: Lucius \& Lucius.

Mak, J. (2003). Informality as an asset? The case of EMU. In T. Christiansen \& S. Piattoni (Hrsg.), Informal governance in the European union (S. 189-206). Cheltenham: Edward Elgar.

McClelland, D. C. (1976). The achieving society. New York: Irvington Publishers.

McNeely, C. L. (1995). Constructing the nation-state. International organization and prescriptive action. Westport, Conn.: Greenwood Press.

Merry, S. E. (2014). Global legal pluralism and the temporality of soft law. The Journal of Legal Pluralism and Unofficial Law 46 (1), 108-122.

Nedergaard, P. (2006). Which Countries Learn from Which? A Comparative Analysis of the Direction of Mutual Learning Processes within the Open Method of Coordination Committees of the European Union and among the Nordic Countries. Cooperation and Conflict 41 (4), 422-442.

Nullmeier, F. (2004). Zwischen Performance und Performanz - Funktionen und Konzepte der Evaluierung in öffentlichen Verwaltungen. In S. Kuhlmann, J. Bogumil \& H. Wollmann (Hrsg.), Leistungsmessung und -vergleich in Politik und Verwaltung. Konzepte und Praxis (S. 47-60). Wiesbaden: VS Verlag für Sozialwissenschaften.

Parsons, T. (1953). A Revised Analytical Approach to the Theory of Social Stratification. In R. Bendix \& S. M. Lipset (Hrsg.), Class, Status and Power. Social stratification in comparative perspective (S. 92-128). New York: Free Press.

Pouliot, V. (2011). Diplomats as Permanent Representatives: The Practical Logics of the Multilateral Pecking Order. International Journal: Canada's Journal of Global Policy Analysis 66 (3), 543-561.

Ravallion, M. (2012). Troubling tradeoffs in the Human Development Index. Journal of Development Economics 99 (2), 201-209.

Rotberg, R. I. (2004). Strengthening governance: Ranking countries would help. The Washington Quarterly 28 (1), 71-81.

Sauder, M. \& Espeland, W. N. (2009). The Discipline of Rankings: Tight Coupling and Organizational Change. American Sociological Review 74 (1), 63-82.

Schmitt, J. (2002). PISA - Reaktionen der teilnehmenden Länder im Spiegel ihrer Presse. Frankreich. Trends in Bildung international 5, 1-6. http://www.pedocs.de/volltexte/ 2012/5213/pdf/tibi_2002_5_Schmitt_Frankreich_D_A.pdf. Zugegriffen 25.04.2016.

Spence, J. (2012). A high price to pay? Britain and the European budget. International Affairs 88 (6), 1237-1260.

Stern, G. (2000). The Structure of International Society: An Introduction to the Study of International Relations. London, New York: Continuum.

Stichweh, R. (2000). Soziologie des Vereins. Strukturbildung zwischen Lokalität und Globalität. In E. Brix \& Richter, R. (Hrsg.), Organisierte Privatinteressen. Vereine in Österreich. Wien: Passagen.

Toulmin, S. (1964). The complexity of scientific choice: A stocktaking. Minerva 2 (3), 343-359.

United Nations Conference on Trade and Development. (2015). The Least Developed Countries Report 2015. Transforming Rural Economies. 
United Nations (2015). General Assembly: Transforming our world: the 2030 Agenda for Sustainable Development. A/RES/70/1 (25. September 2015).

United Nations (2016). General Assembly: Graduation of Angola from the least developed country category. A/RES/70/253 (10. März 2016).

van der Vleuten, A. \& Verloo, M. (2012). Ranking and benchmarking: The political logic of new regulatory instruments in the fields of gender equality and anti-corruption. Policy \& Politics 40 (1), 71-86.

Werron, T. (2012). Wie ist globale Konkurrenz möglich? Zur sozialen Konstruktion globaler Konkurrenz am Beispiel des Human Development Index. Soziale Systeme 18 $(1+2), 168-203$.

Wimmer, H. (2000). Die Modernisierung politischer Systeme. Staat - Parteien Öffentlichkeit. Wien: Böhlau.

World Bank (2016). World Development Indicators 2016. Washington, DC: World Bank. 